\title{
RIESGO BIOLÓGICO GENERADO POR EL MANEJO DE RESIDUOS SÓLIDOS EN UN CENTRO HOSPITALARIO
}

\author{
Álvaro Quintana Salcedo ${ }^{1 *}$, Carolin Ramos De La Espriella ${ }^{2}$ y Edgar Lugo Calderón ${ }^{3}$ \\ ${ }_{1,2,3}$ Corporación Universitaria Rafael Núñez - CURN. Cartagena de Indias, Colombia \\ *Autor de correspondencia: alvaro.quintana@ curnvirtual.edu.co
}

Recibido Febrero 2019; Aceptado Mayo 2019

\begin{abstract}
Resumen - Dentro de las instituciones de salud y similares, el personal de servicios generales en el desarrollo de sus actividades, se encuentra expuesto a agentes biológicos patógenos que puede afectar el normal funcionamiento de los diferentes órganos y sistemas del cuerpo humano, lo cual se evidencia en la aparición de cuadros infecciosos que pueden comprometer la salud e incluso la vida. El objetivo de este articulo es evaluar el riesgo biológico generado por el manejo de residuos sólidos por parte del personal de servicios generales en la E.S.E Hospital Local Cartagena de Indias. Se realizó un estudio descriptivo de enfoque cuantitativo. Se utilizó la matriz de riesgo de la guía técnica colombiana 45 y la encuesta de 14 ítems basada en el Sistema de Vigilancia Epidemiológica para el Riesgo Biológico (modelo para el control del riesgo biológico) de la ARL SURA aplicado al sector hospitalario. La muestra fue de 34 trabajadoras del área de servicios generales, distribuidos en los CAP intraurbanos de la E.S.E. Cartagena. Los resultados muestran que las actividades donde presenta mayor contacto con agentes biológicos son en el lavado de camillas, cambio de bolsas rojas para residuos peligrosos y lavado de guardián (se observó durante el desarrollo de la investigación), siendo esta última inusual. Se concluye que las medidas preventivas y de seguridad que adopta el personal en su puesto de trabajo son eficientes en relación a tener su área limpia y en orden, son conscientes que al realizar sus actividades entran en contacto con agentes biológicos. Por ello se deben realizar capacitaciones de autocuidado, capacitación e inspección del uso y mantenimiento de los elementos de protección personal, así mejorando las acciones preventivas evitando accidentes o enfermedades laborales.
\end{abstract}

Palabras clave: Riesgo biológico, medidas preventivas, accidente de trabajo, enfermedad laboral, seguridad y salud en el trabajo.

\section{BIOLOGICAL RISK GENERATED BY THE MANAGEMENT OF SOLID WASTE IN A HOSPITAL}

\begin{abstract}
Within health and similar institutions, general service personnel in the development of their activities, are exposed to pathogenic biological agents that can affect the normal functioning of different organs and systems of the human body, which is evidenced in the emergence of infectious conditions that can compromise health and even life. The objective of this article is to evaluate the biological risk generated by the management of solid waste by general service personnel at the E.S.E. Hospital Local Cartagena de Indias. A descriptive study with a quantitative approach was conducted. The risk matrix of Colombian technical guide 45 and the 14-item survey based on the Epidemiological Surveillance System for Biological Risk (model for the control of biological risk) of ARL SURA applied to the hospital sector were used. The sample was 34 workers from the general services area, distributed in the intra-urban KAPs of the E.S.E. Cartagena. The results show that the activities where there is the greatest contact with biological agents are in washing stretchers, changing red bags for hazardous waste and guard washing (this was observed during the development of the research), the latter being unusual. It is concluded that the preventive and security measures adopted by the personnel in their workplace are efficient in relation to having their area clean and in order, they are
\end{abstract}

IPSA SCIENTIA - Vol. 4 N 1 - 2019 


\section{Riesgo biológico generado por el manejo de residuos sólidos en un centro hospitalario}

aware that when carrying out their activities they come into contact with biological agents. Therefore, self-care training, training and inspection of the use and maintenance of personal protection elements must be carried out, thus improving preventive actions by avoiding accidents or occupational diseases.

Keywords: Biological risk, preventive measures, accidents at work, occupational diseases, safety and health at work.

\section{Introducción}

Prevenir la enfermedad en el área laboral y promover la salud del trabajador es una disciplina que viene en auge por ley y que centra sus objetivos en promocionar el bienestar del capital humano de las empresas. No escapan de esa influencia, las instituciones que prestan servicios de salud. Prevenir los accidentes del trabajo y las enfermedades profesionales, proveer un ambiente laboral seguro, que mejore las condiciones físicas, mentales y sociales del trabajador, es un derecho y debe asegurarse desde todos los medios, la seguridad y salud en el trabajo como actividad multidisciplinaria se encarga de esto (Bravo \& Díaz, 2016; Huanca et al., 2016; Leitner \& Resch, 2005).

Dentro de las instituciones de salud y similares, el personal de servicios generales en el desarrollo de sus actividades, se encuentra expuesto a agentes biológicos patógenos que afecta el normal funcionamiento de los órganos y sistemas del cuerpo humano, lo cual se evidencia en la aparición de cuadros infecciosos que pueden comprometer los pulmones, el corazón, los riñones, las articulaciones, el aparato digestivo, los ojos, entre otros. Razón por la cual la SST tiene una gran relevancia, ya que compromete a toda la organización, a generar y promover el trabajo sano, seguro, buenos ambientes y organizaciones de trabajo; aumentar el bienestar físico, mental y social de los trabajadores, respaldar el perfeccionamiento y el mantenimiento de su capacidad de trabajo (Romero et al., 2007; Marrugo Ligardo, 2017; Jaimes Morales, 2018).

El contacto con agentes biológicos requiere de la utilización de elementos de protección, herramientas de trabajo adecuado y de una infraestructura que cumpla con las normas de bioseguridad según su clasificación del peligro al que está expuesto, además de una valoración de riesgo biológico con el cual se pueda gestionar un trabajo seguro y saludable en el ambiente de trabajo (Pimentel et al., 2007; Neveu \& Matus, 2007; Orozco, 2013; Lengua et al., 2014). Es por ello que se realiza esta investigación, buscando estudiar los riesgos biológicos a los cuales están expuestos estos trabajadores de servicios generales, conocer las estrategias prevención que usan las entidades prestadoras de salud frente a estos agentes biológicos, con el fin de proponer recomendaciones que ayuden a mejorar la situación actual, prevenir accidentes y algún tipo de enfermedad laboral (Hernández et al., 2006; Suárez \& Junco, 2012; Cottin et al., 2016; Palacio Angulo et al., 2018).

\section{Métodos}

El presente estudio se enmarca dentro de los estudios de tipo descriptivo de enfoque cuantitativo, el cual ayudara a valorar los riesgos biológicos con relación a los agentes biológicos a los cuales se ven expuestos los trabajadores; además ayudará a conocer las medidas preventivas que utiliza el personal de servicios generales para el correcto manejo de residuos peligros con agentes biológicos. La población la constituye el personal de servicios generales que labora en los Centros de Atención Primaria (CAP) urbanos de la E.S.E Hospital Local Cartagena de Indias. La cual está comprendida por 34 Trabajadores.

IPSA SCIENTIA - Vol. 4 Nº $1-2019$ 


\section{Riesgo biológico generado por el manejo de residuos sólidos en un centro hospitalario}

Se realizó un muestreo con el total de la población de trabajadores por ser relativamente pequeña la población total, es decir los 34 trabajadores que pertenecen al área de servicios generales, distribuidas en los CAP intraurbanos de la ciudad. Los CAP que serán intervenidos son 7 CAP: Arroz Barato, Blas de Lezo, Canapote, La Esperanza, Nuevo Bosque, Olaya y Pozón.

En la toma de datos para la valoración de riesgo biológico, se utilizó la matriz de riesgo de la guía técnica colombiana 45 , que ofrece una serie de pasos como la identificación de peligros a los cuales están expuestos los empleados y busca dar un diagnóstico de las condiciones de trabajo; establece los puntos críticos de peligro donde existe un potencial para la presencia de los accidentes de trabajo y/o generación de enfermedades que podrían ocurrir a corto o mediano plazo en desarrollo de las actividades y la valoración del riesgo, indicando las situaciones de peligro biológico que pueden generar pérdidas materiales o humanas (Tapias-Vargas et al., 2010).

Para conocer y obtener información sobre las medidas preventivas y de seguridad adoptada por el personal de servicios generales, se usó el instrumento de recolección de información del Sistema de Vigilancia Epidemiológica para el Riesgo Biológico (modelo para el control del riesgo biológico) de la ARL SURA aplicado al sector hospitalario. El presente trabajo se llevó a cabo por medio de una serie de etapas consecutivas, las cuales ayudaran al logro de los objetivos planeados. A continuación se presenta un paso a paso para llevar a cabo la investigación:

1. Búsqueda bibliográfica.

2. Construcción del documento.

3. Solicitud de permiso a la institución prestadora de servicio de salud.

4. Socialización de la investigación.

5. Cronograma de actividades para implementar la matriz y aplicar la encuesta.

6. Tabulación de los datos.

7. Análisis y Discusión de los resultados.

8. Recomendaciones.

\section{Resultados}

Los resultados obtenidos a través de los instrumentos de recolección, se presentan a continuación por medio de tablas y gráficos con su análisis descriptivo.

La muestra final constituyó un número de 34 trabajadores de servicios generales que labora en los centros de atención primaria de la E.S.E. Hospital Local Cartagena de Indias.

A continuación se muestra la valoración del riesgo de las actividades en las que el personal de servicios generales diariamente está más expuesto al riesgo biológico.

Con relación a los datos aportados por el cuadro 1, se observan los siguientes resultados dentro de la actividad de limpieza del guardián 
Riesgo biológico generado por el manejo de residuos sólidos en un centro hospitalario

\begin{tabular}{|c|c|c|c|c|c|c|c|c|}
\hline \multirow[b]{2}{*}{ Actividad } & \multicolumn{7}{|c|}{ Evaluación del riesgo } & \multirow{2}{*}{$\begin{array}{l}\text { Valoración del riesgo } \\
\text { Aceptabilidad del riesgo }\end{array}$} \\
\hline & $\begin{array}{c}\text { Nivel de } \\
\text { deficiencia }\end{array}$ & $\begin{array}{l}\text { Nivel de } \\
\text { exposición }\end{array}$ & $\begin{array}{c}\text { Nivel de } \\
\text { probabili- } \\
\text { dad } \\
\text { (NDxNE) }\end{array}$ & $\begin{array}{l}\text { Interpreta- } \\
\text { ción del } \\
\text { nivel de } \\
\text { probabill- } \\
\text { dad }\end{array}$ & $\begin{array}{c}\text { Nivel de } \\
\text { consecuen-cia }\end{array}$ & $\begin{array}{l}\text { Nivel del } \\
\text { riesgo e } \\
\text { intervén- } \\
\text { ción }\end{array}$ & $\begin{array}{l}\text { Interpreta- } \\
\text { ción del } \\
\text { NR }\end{array}$ & \\
\hline $\begin{array}{l}\text { Limpieza de } \\
\text { guardián }\end{array}$ & 10 & 2 & 20 & Alto & 25 & 500 & II & $\begin{array}{l}\text { Aceptable o no aceptable } \\
\text { con control especifico }\end{array}$ \\
\hline $\begin{array}{l}\text { Cambio de bolsa de } \\
\text { residuos biológicos }\end{array}$ & 6 & 3 & 18 & Alto & 25 & 450 & II & $\begin{array}{l}\text { Aceptable con control o no } \\
\text { aceptable }\end{array}$ \\
\hline Lavado de camilla & 6 & 3 & 18 & Alto & 25 & 450 & II & $\begin{array}{l}\text { Aceptable con control o no } \\
\text { aceptable }\end{array}$ \\
\hline
\end{tabular}

- Interpretación del nivel de deficiencia: Al analizar el nivel de deficiencia como lo muestra el cuadro 1. Se evidencio que el personal al realizar esta actividad se expone a agentes biológicos, elementos corto punzantes, que son posibles generadores de incidentes y accidentes muy significativos. Lo cual se interpreta su nivel de defíciencia en 10.

- Interpretación del nivel de exposición: En esta actividad, el nivel de exposición es ocasional, donde la situación de exposición se presenta alguna vez durante su jornada laboral y por un periodo de tiempo corto. Lo cual su valor es de 2 .

- Interpretación del nivel de probabilidad: Al analizar el nivel de probabilidad de esta actividad, se evidencia que la probabilidad es alta, ya que una situación deficiente con una exposición ocasional, puede hacer que la materialización del riesgo biológico suceda varias veces en la vida laboral.

- Interpretación del nivel de consecuencia: Este nivel es grave (25), ya que al realizar esta actividad genera lesiones o enfermedades con incapacidad laboral temporal.

- Interpretación del nivel de riesgo: El nivel de riesgo en esta actividad es de II, con un valor de 500, por lo cual tiene que ser corregido y adoptar medidas de control inmediatas, donde nos da una valoración de riesgo aceptable con controles, pero a la vez no aceptable.

Con respecto a las actividades de cambio de bolsa de residuos biológicos y lavado de camilla, se encontraron para ambos casos los siguientes resultados:

- Interpretación del nivel de defíciencia: Al analizar el nivel de deficiencia, se evidencio que el personal al realizar estas actividades se expone a agentes biológicos que pueden dar lugar a consecuencias significativas. Lo cual se interpreta su nivel de deficiencia en 6.

- Interpretación nivel de exposición: El nivel de exposición en estas actividades es frecuente, donde la situación de exposición se presenta varias veces durante la jornada laboral por tiempos cortos. Lo cual su valor es de 3 .

- Interpretación del nivel de probabilidad: Al analizar el nivel de probabilidad de estas actividades, se evidencia que la probabilidad es alta, ya que una situación deficiente con una exposición ocasional, puede hacer que la materialización del riesgo biológico suceda varias veces en a vida laboral.

IPSA SCIENTIA - Vol. 4 N 1 - 2019 
- Interpretación del nivel de consecuencia: Este nivel es grave (25), ya que al realizar estas actividades generan lesiones o enfermedades con incapacidad laboral temporal.

- Interpretación del nivel de riesgo: El nivel de riesgo en estas actividades es de II. Con un valor de 450, por lo cual tiene que ser corregido y adoptar medidas de control inmediatas, donde nos da una valoración de riesgo aceptable con controles, pero a la vez no aceptable.

Al aplicar el instrumento "Encuesta de medidas preventivas y de seguridad", y al tabular y procesar los datos, se muestran a continuación los principales resultados: del total de trabajadores luego de explicarles el estudio y presentarles el consentimiento, un número de 8 trabajadores se abstuvieron de participar, por ello solo se encuestaron 26 trabajadores.

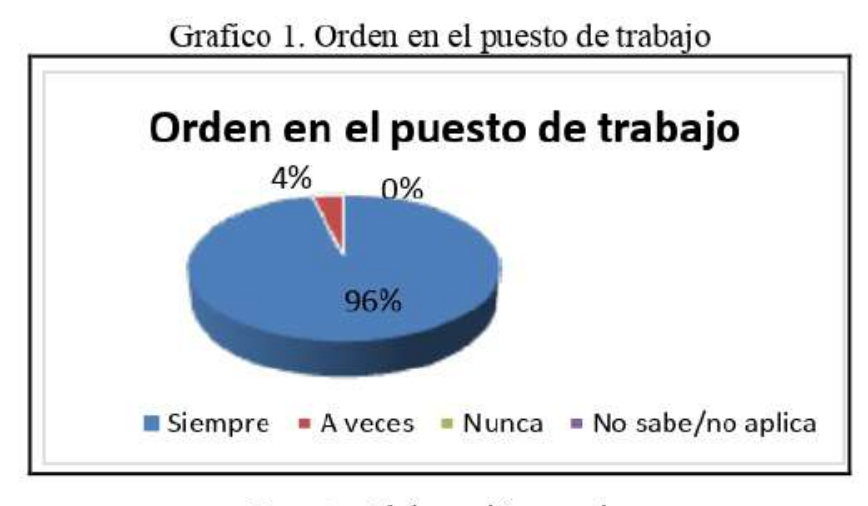

Fuente: Elaboración propia

Entre las medidas preventivas y de seguridad adoptadas por el personal de servicios generales, se evidencia que, tal como se observa en el gráfico 1, al indagar sobre el orden en el puesto de trabajo, para el $96 \%$ (25) de los encuestados, siempre se mantiene ordenado y limpio, para el $4 \%$ (1) restante a veces se mantiene ordenado y limpio.

Grafico 2. Contacto con agentes biológicos durante los procedimientos

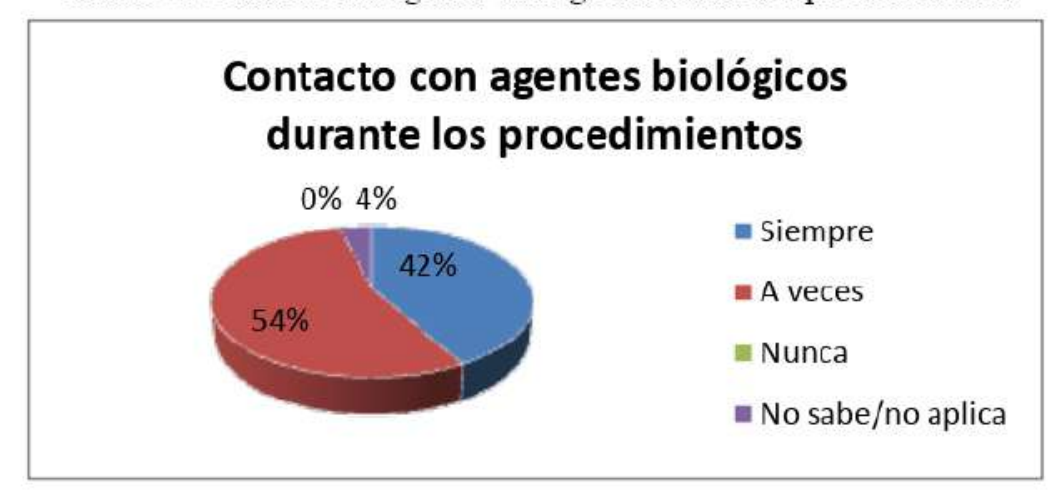

Fuente: Elaboración propia

Según el gráfico 2, el 42\% (11) de los trabajadores respondió que siempre se encuentran en contacto con agentes biológicos durante la realización de los procedimientos, el 54\% (14) respondió que solo a veces, mientras que el $4 \%$ (1) restantes respondió que no sabe. 
Riesgo biológico generado por el manejo de residuos sólidos en un centro hospitalario

Grafico 3. Elementos de protección personal

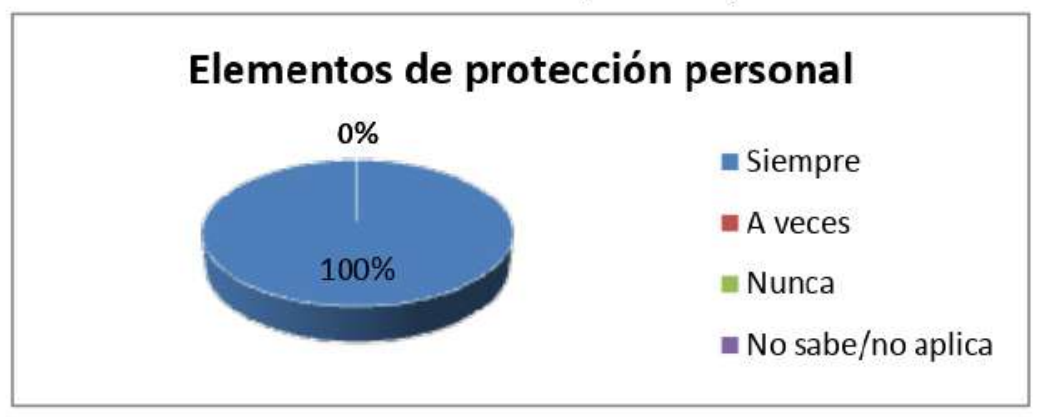

Fuente: Elaboración propia

De acuerdo con el gráfico 3, el 100\% (26) de los trabajadores encuestados respondió que cuentan con los elementos de protección personal.

Grafico 4. Áreas de descontaminación y lavado

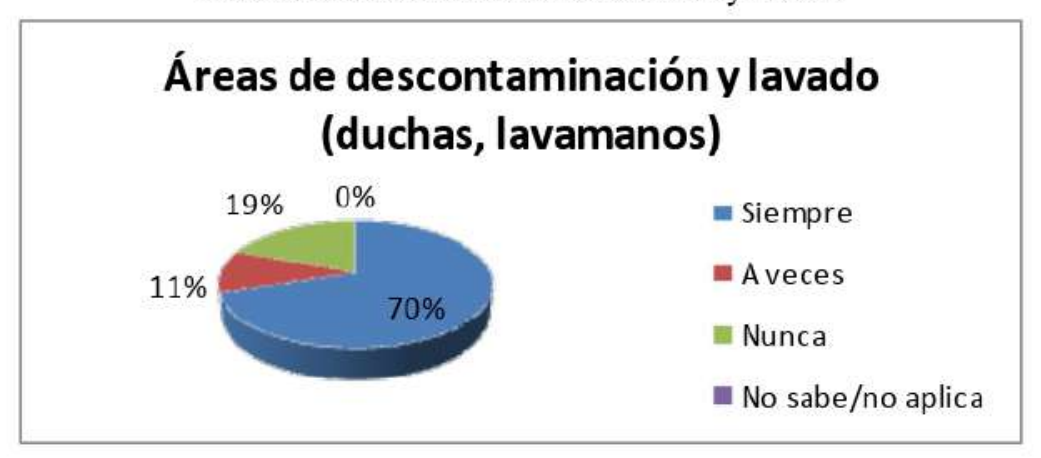

Fuente: Elaboración propia

En el gráfico 4 se observa que el 70\% (18) de los trabajadores respondió que si cuentan con áreas de descontaminación y lavado, el 19\% (5) que estas no existen, mientras que el 11\% (3) restante respondió que a veces no se encuentran disponibles las áreas de lavado.

Grafico 5. Ducharse antes de abandonar el área de trabajo

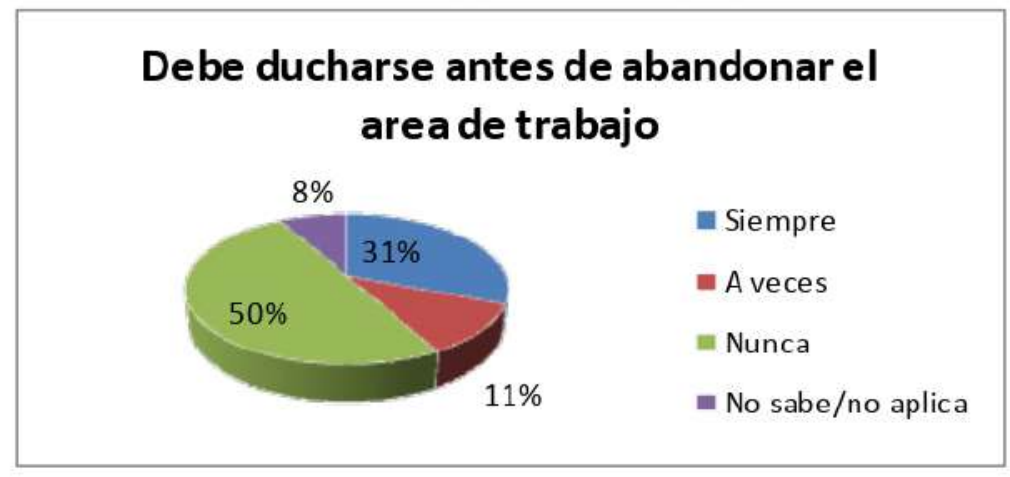

Fuente<: Elaboración propia 
Riesgo biológico generado por el manejo de residuos sólidos en un centro hospitalario

Según el gráfico 5, el 50\% (13) de los trabajadores respondió que no se duchan antes de abandonar el trabajo, el 31\% (8) afirma que siempre se ducha antes de abandonar el área de trabajo, el 11\% (3) que a veces y el $8 \%$ (2) restantes no sabe.

Grafico 6. Vacuna contra la hepatitis

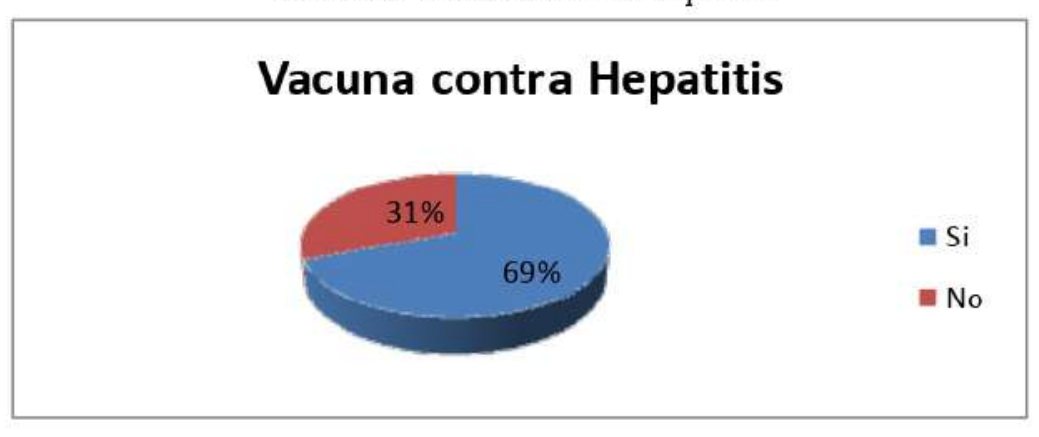

Fuente: Elaboración propia

De acuerdo con el gráfico 6, el 69\% (18) de los trabajadores respondió que si recibió la vacuna de hepatitis mientras que el $31 \%$ (8) no la tiene.

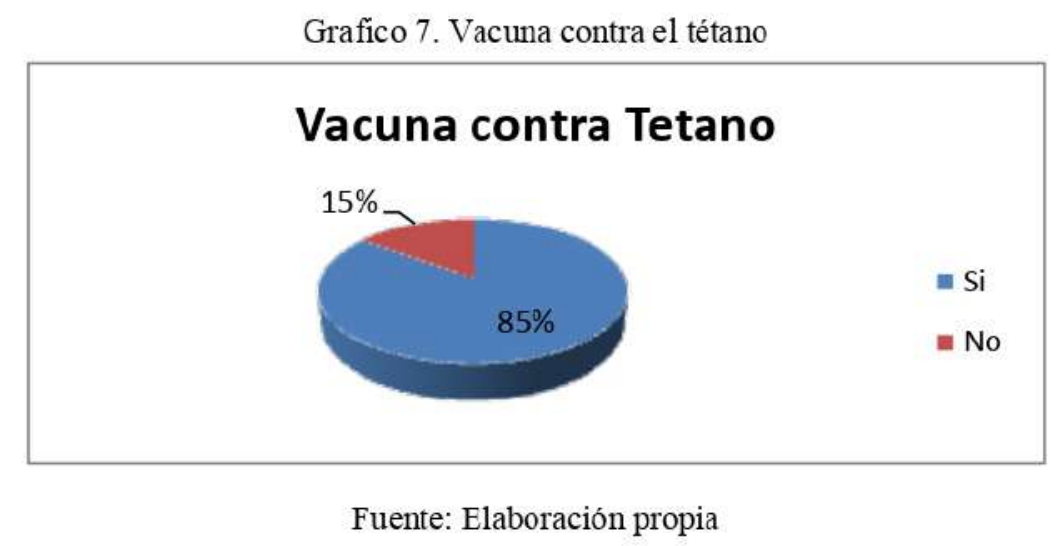

En el gráfico 7 se observa que el $85 \%$ (22) de los trabajadores respondió que si recibió la vacuna del tétano, mientras que el $15 \%$ (4) respondió que no la tiene.

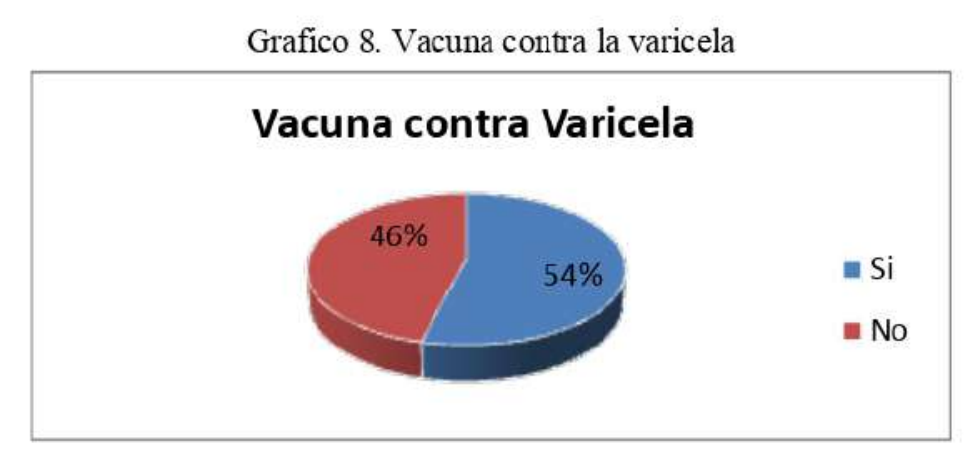

Fuente: Elaboración propia 
Riesgo biológico generado por el manejo de residuos sólidos en un centro hospitalario

Según el gráfico 8 , el $54 \%$ (14) de los trabajadores respondió que sí recibido la vacuna contra la varicela y el $46 \%$ (12) restante respondió que no la tiene.

Grafico 9. Vacuna contra el sarampión

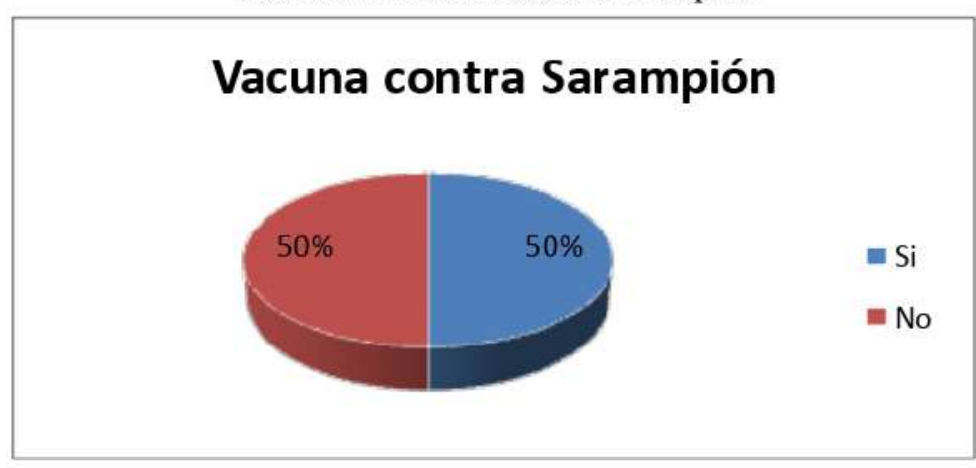

Fuente: Elaboración propia

En el gráfico 9 se evidencia que el 50\% (13) de los trabajadores respondió que si recibió la vacuna contra el sarampión, mientras que el otro $50 \%(13)$ no la tiene.

Grafico 10. Vacuna contra la rubeola

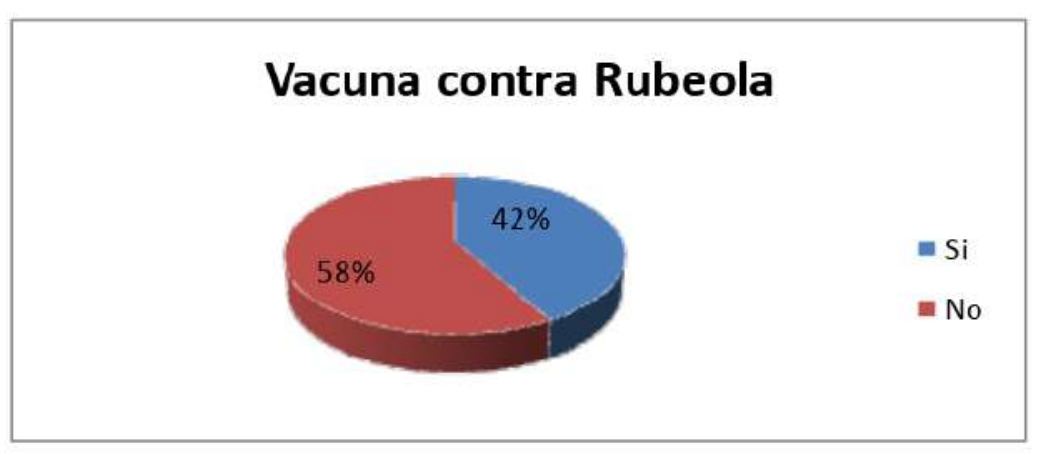

Fuente: Elaboración propia

Los resultados aportados por el gráfico 10 muestran que el 58\% (15) de los trabajadores respondió que si recibió la vacuna contra la rubeola mientras que el $42 \%$ (11) respondió que no la tiene.

Grafico 11. Vacuna contra la difteria

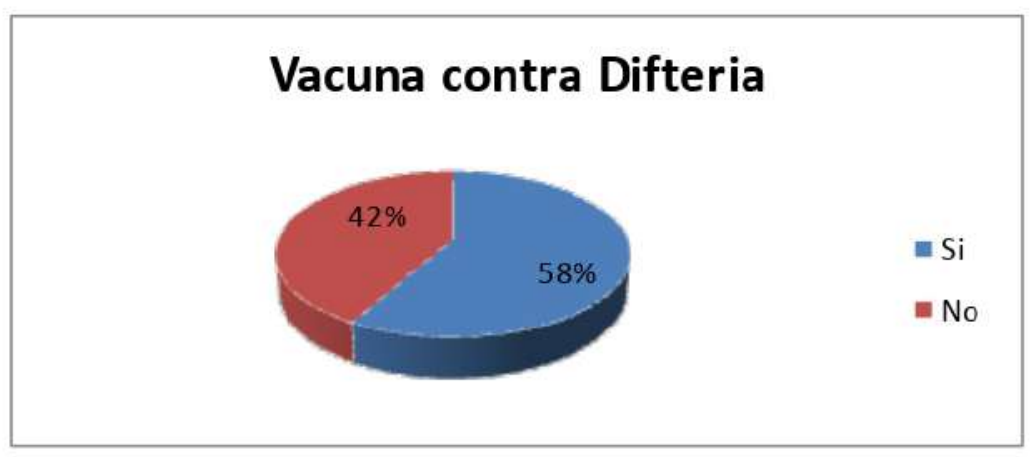

Fuente: Elaboración propia

IPSA SCIENTIA - Vol. 4 No 1 - 2019 
Riesgo biológico generado por el manejo de residuos sólidos en un centro hospitalario

En el gráfico 11 se muestra que el 58\% (15) de los trabajadores respondió que si recibió la vacuna contra la difteria mientras que el $42 \%$ (11) respondió que no la tiene.

Grafico 12. Accidentes con agentes biológicos

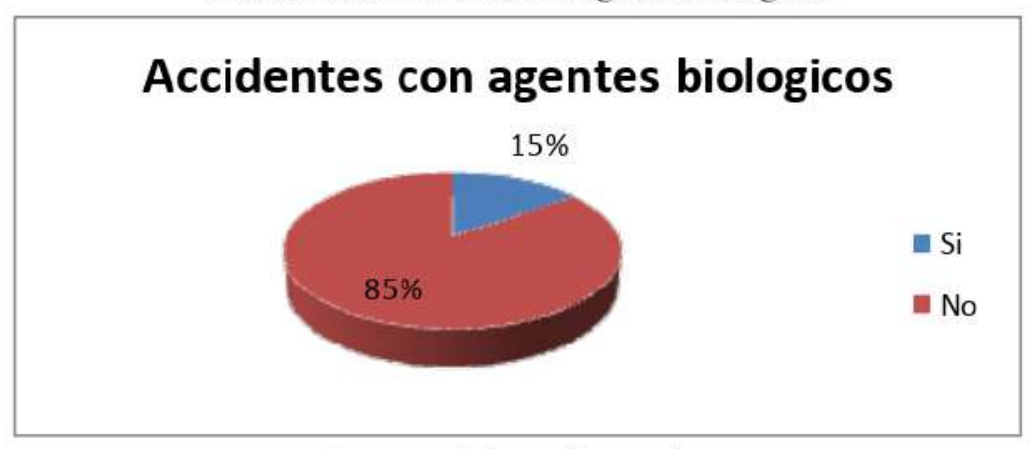

Fuente: Elaboración propia

A la pregunta si ha sufrido accidentes con agentes biológicos en su trabajo, los trabajadores respondieron que en un $85 \%$ (22) no han recibido ningún tipo de accidente y el $15 \%$ (4) afirma que si ha sufrido accidentes los cuales son: accidente con peróxido de sodio, pinchazo con aguja y con ampolla, tal como se muestra en el gráfico 12 .

Grafico 13. Antecedentes de gastroenteritis - diarrea

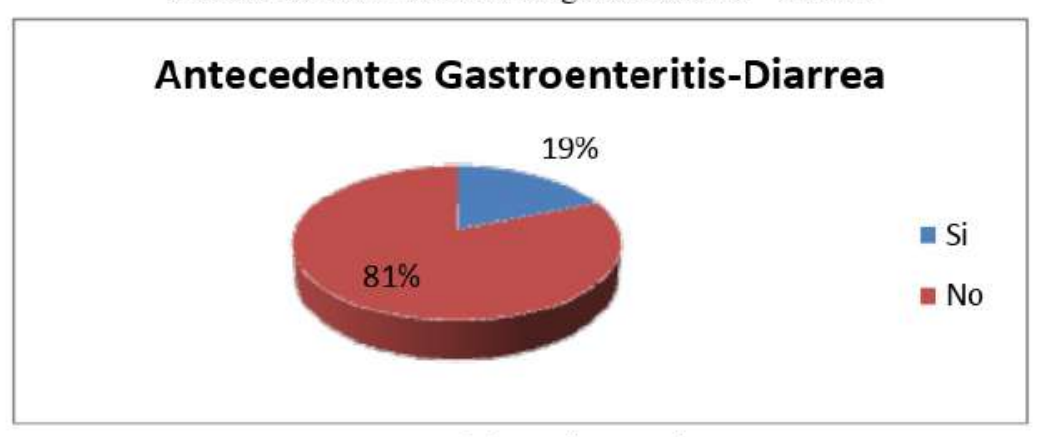

Fuente: Elaboración propia

Se observa en el gráfico 13, que de los 26 trabajadores encuestados, el $81 \%$ (21) respondió no haber sufrido esta patología mientras que el 19\% (5) si la ha padecido.

Grafico 14. Antecedentes de infecciones cutáneas

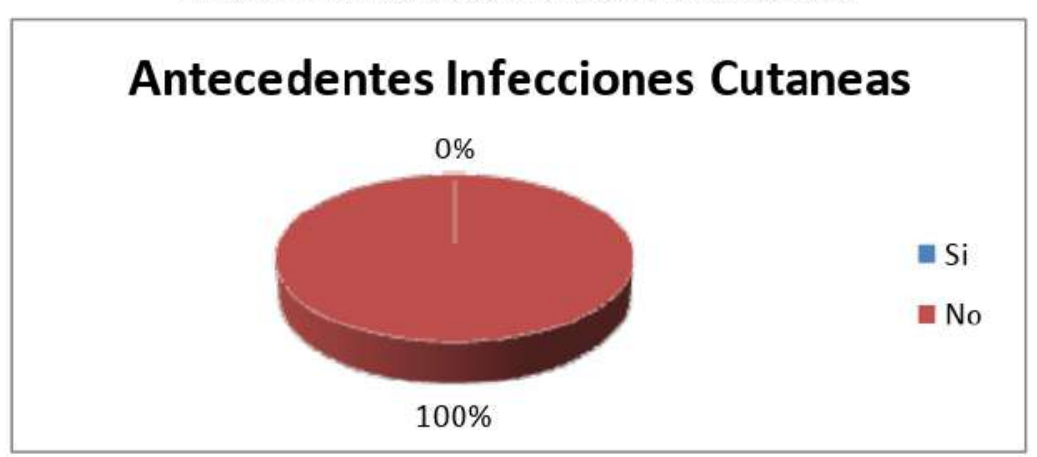

Fuente: Elaboración propia

IPSA SCIENTIA - Vol. 4 No 1 - 2019 
Riesgo biológico generado por el manejo de residuos sólidos en un centro hospitalario

Con relación al indicador correspondiente al gráfico 14, el 100\% (26) de los encuestados negó haber sufrido esta patología.

Grafico 15. Antecedentes de alergias

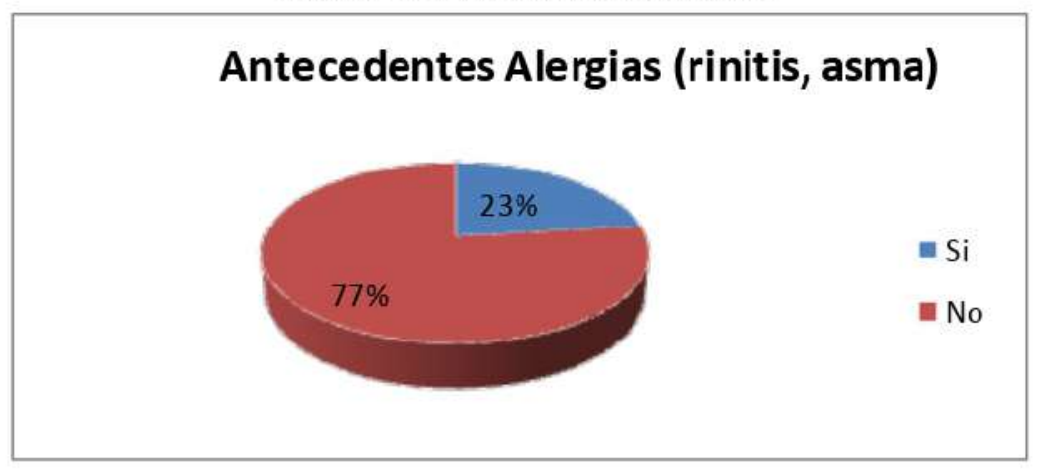

Fuente: Elaboración propia

A la pregunta si ha sufrido esta patología el $77 \%$ (20) negó haberla padecido mientras que el 23\% (6) afirmo haberla tenido, tal como se observa en el gráfico 15.

Grafico 16. Antecedentes de otitis

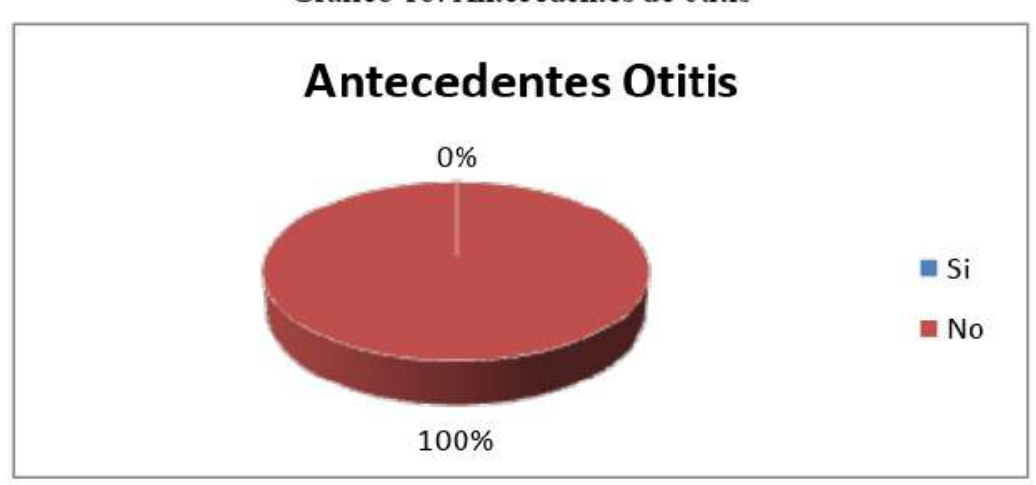

Fuente: Elaboración propia

El 100\% (26) de los encuestados negó haber sufrido esta patología; siendo estos resultados ilustrados en el gráfico 16.

Grafico 17. Antecedentes de conjuntivitis

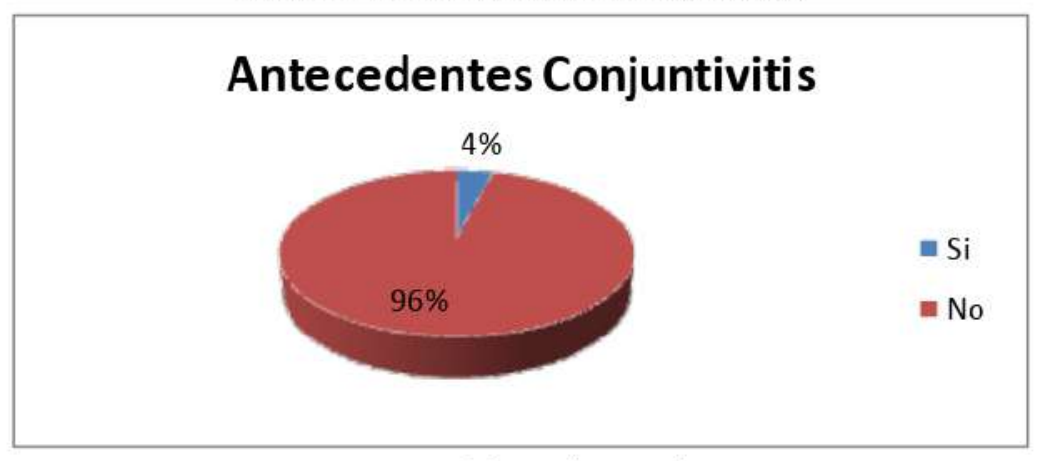

Fuente: Elaboración propia

IPSA SCIENTIA - Vol. 4 No 1 - 2019 
Riesgo biológico generado por el manejo de residuos sólidos en un centro hospitalario

A la pregunta si ha sufrido esta patología el 96\% (25) negó haberla padecido mientras que el 4\% (1) afirmo haberla tenido, resultados aportados por el gráfico 17.

Grafico 18. Antecedentes de resfriados

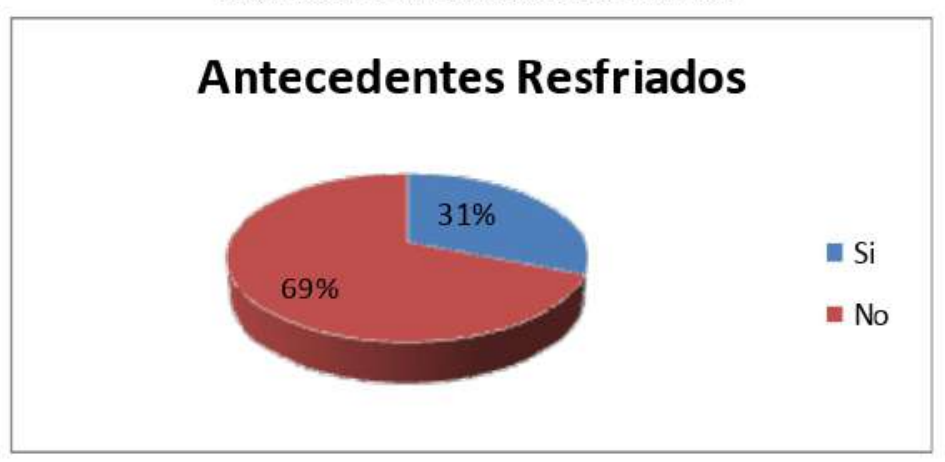

Fuente: Elaboración propia

El gráfico 18 muestra las respuestas a la pregunta si ha sufrido esta patología, el 69\% (18) negó haberla padecido mientras que el $31 \%$ (8) afirmó haberla tenido.

Grafico 19. Antecedentes de neumonía

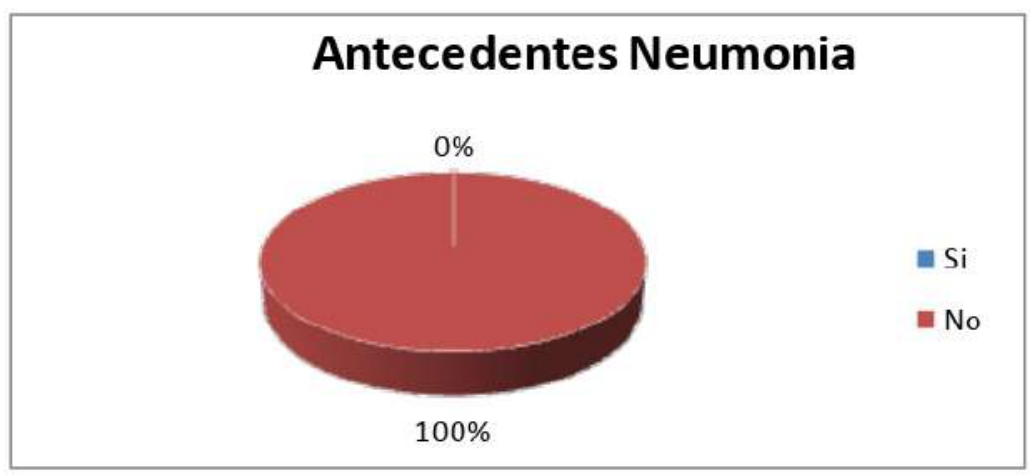

Fuente: Elaboración propia

Como respuesta a la pregunta realizada, los 26 trabajadores que representa el $100 \%$ negó haber sufrido esta patología, tal como se muestra en el gráfico 19.

Grafico 20. Manual de bioseguridad y procedimiento

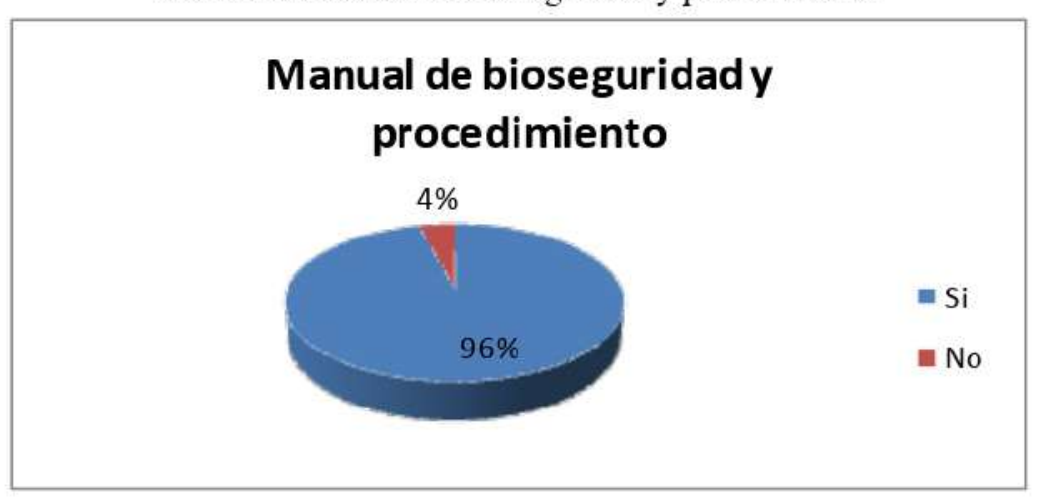

Fuente: Elaboración propia

IPSA SCIENTIA - Vol. 4 Nº 1 - 2019 
Riesgo biológico generado por el manejo de residuos sólidos en un centro hospitalario

A la pregunta, cuenta con un manual de bioseguridad y procedimiento, el $96 \%$ (25) de los encuestaron afirmaron tenerlo mientras que el 4\% (1) restante lo negó; estas cifras se evidencian en el gráfíco 20.

Grafico 21. Capacitaciones sobre el uso, cuidados y mantenimiento de los EPP

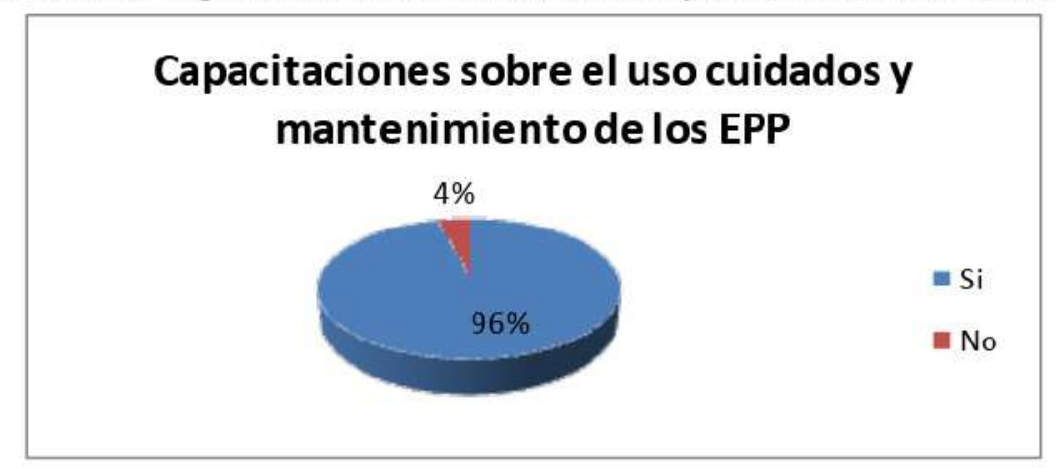

Fuente: Elaboración propia

Según el gráfico 21, el 96\% (25) de los trabajadores afirmó haber recibido capacitaciones sobre el uso cuidados y mantenimiento de los elementos de protección personal (EPP) mientras que el 4\% (1) lo negó.

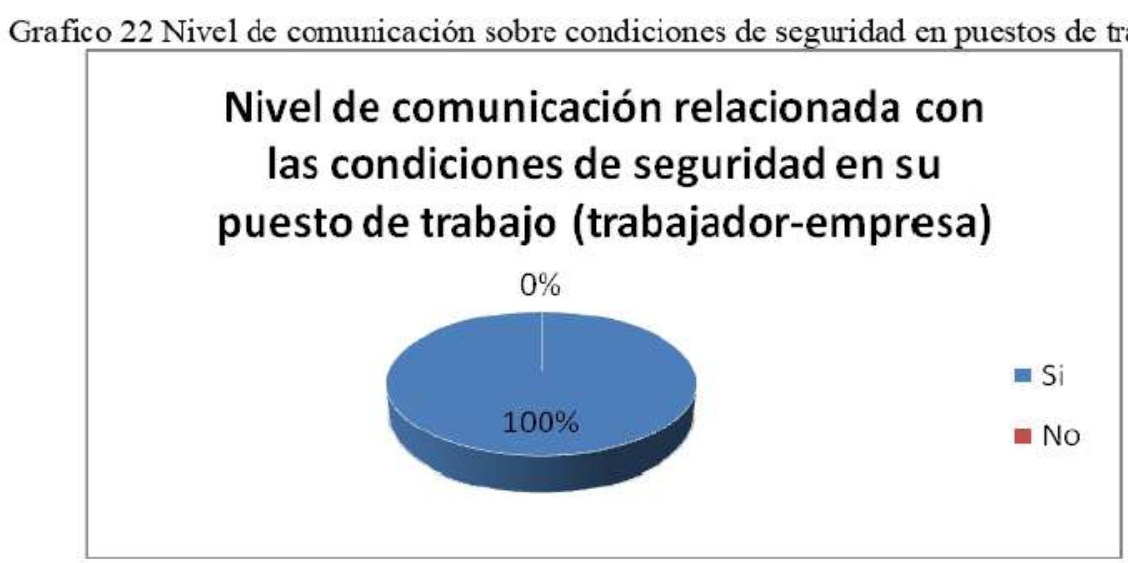

Fuente: Elaboración propia

El 100\% (26) de los encuestados indica que si existe un buen nivel de comunicación de la empresa con los trabajadores, según cifras aportadas por el gráfico 22

Grafíco 23. Estándares, protocolos, guías o procedimientos de bioseguridad

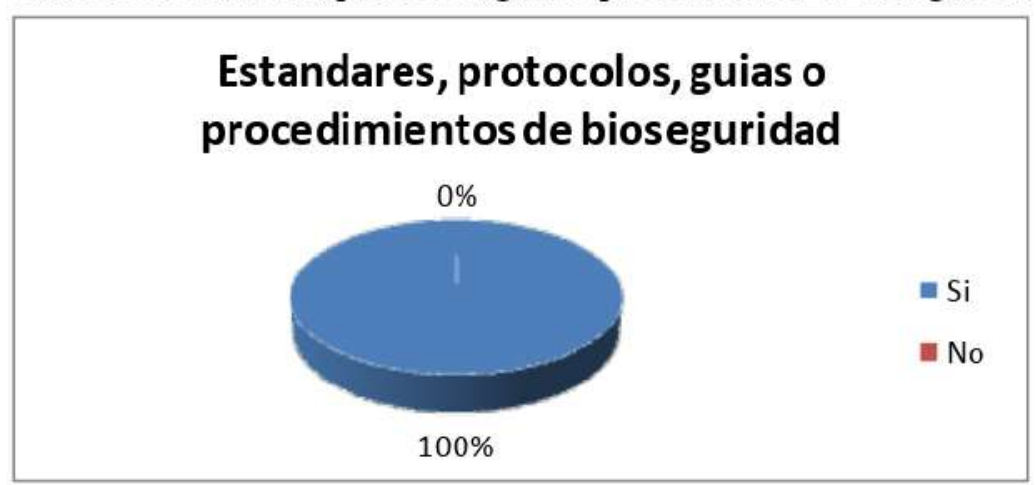

Fuente: Elaboración propia

IPSA SCIENTIA - Vol. 4 No 1 - 2019 
Riesgo biológico generado por el manejo de residuos sólidos en un centro hospitalario

El $100 \%$ (26) de los encuestados afirmó que cumplen con los protocolos de bioseguridad, tal como se observa en el gráfico 23 .

Grafico 24. Uso, mantenimiento y almacenamiento de los EPP

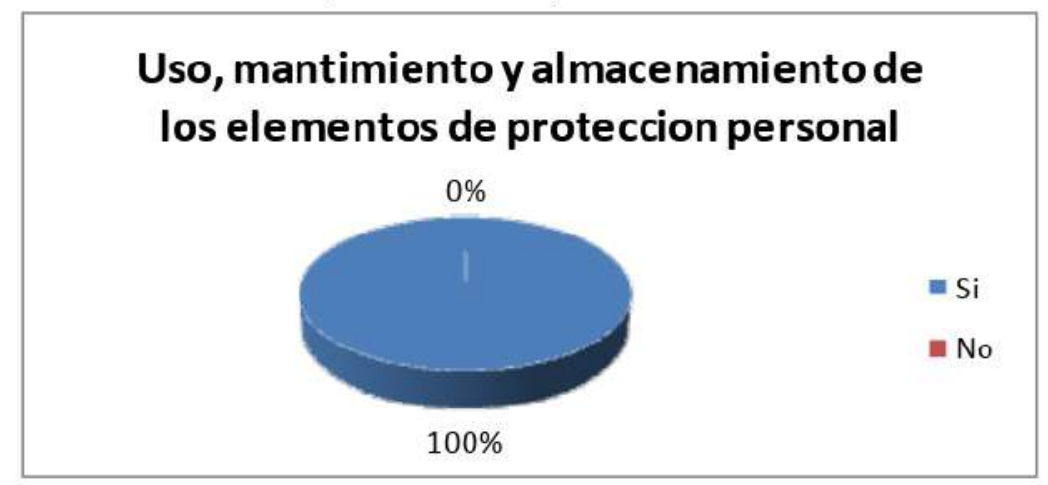

Fuente: Elaboración propia

El $100 \%$ (26) de los encuestados afirmo que usa mantiene y almacena los elementos de protección personal (EPP) asignados para su oficio., tal como se muestra en el gráfico 24 .

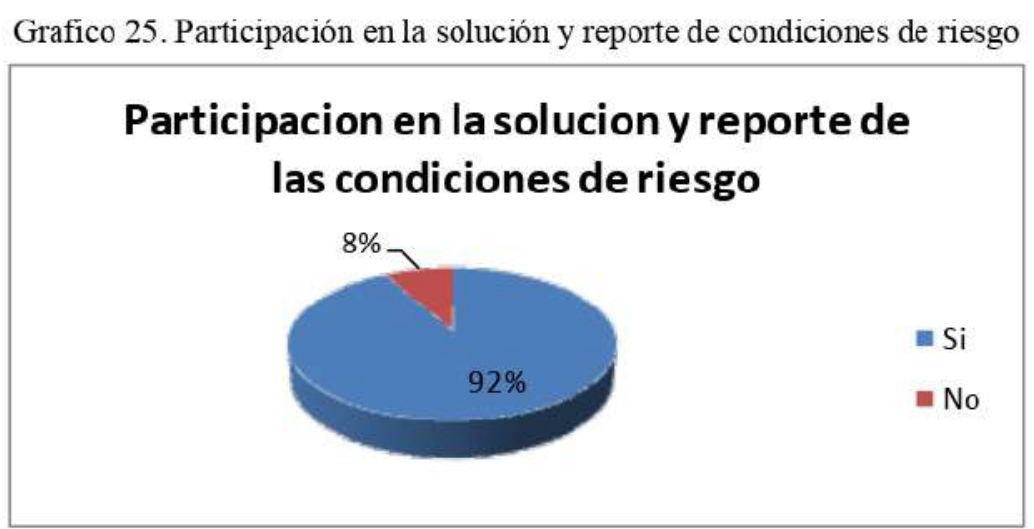

Fuente: Elaboración propia

Finalmente, según cifras aportadas por el gráfico 25 , el $92 \%$ (24) de los encuestados afirmo haber participado en la solución y reporte de las condiciones de riesgo en su puesto de trabajo, mientras que el $8 \%$ (2) lo negó.

\section{Conclusiones}

De los resultados presentados anteriormente, se concluye que:

Las actividades donde presenta mayor contacto con agentes biológicos son en el lavado de camillas, cambio de bolsas rojas para residuos peligrosos y lavado de guardián (se observó durante el desarrollo de la investigación), siendo esta última inusual (se evidencio un accidente), ya que los guardianes son recipientes desechables y luego de utilizarse deben sellarse, depositarse en una bolsa roja y ser rotulados como residuos peligrosos. 


\section{Riesgo biológico generado por el manejo de residuos sólidos en un centro hospitalario}

Estas actividades deben desarrollarse bajo medidas de control, el personal debe contar con los elementos de protección personal adecuados para cada labor. Ya que no hay una correcta capacitación e inspección del uso y mantenimiento de los elementos de protección personal (ver anexo).

Las medidas preventivas y de seguridad que adopta el personal en su puesto de trabajo son eficientes en relación a tener su área limpia y en orden, son conscientes que al realizar sus actividades entran en contacto con agentes biológicos; Algo que cabe añadir es que el personal aun sabiendo que deben ducharse no lo pueden realizar debido que en puesto de trabajo no cuentan con duchas.

Las medidas preventivitas que adoptan en respecto a las condiciones de salud, se supo que al personal le han aplicado algunas vacunas, como por ejemplo contra el tétano, hepatitis B; no todas cuentan con las vacunas al día, aun así están laborando y diariamente exponiéndose a agentes biológicos.

De acuerdo a los resultados, se evidencia que la empresa les suministra los manuales de bioseguridad y procedimientos, reciben capacitaciones sobre el uso, cuidados y mantenimiento de los elementos de protección personal, manifiestan tener buena comunicación con la gerencia con relación a las condiciones de seguridad; que cumplen con los estándares, protocolos y guías de bioseguridad; que usan, mantienen y almacenan correctamente los elementos de protección personal, cabe decir que donde ellas guardan sus elementos y dejan sus bolsos se lavan las canecas de rojas de residuos peligrosos.

\section{Referencias}

Bravo Martín, S.F. \& Díaz Morales, D. (2016). Riesgo biológico en Instituciones de salud: control y precauciones en la atención a pacientes. Medicentro Electrónica, 20(2), 153-155.

Cottin, I., Vallery, G. \& Dahak, S. (2016). Uso situado de los EPP (equipos de protección personal) frente al riesgo biológico: Ejemplo de un laboratorio seguro de contención de nivel 3. Laboreal, 12(2), 56-74.

Hernández Valdez, E., Acosta González, M., Nadal Tur, B., Pijuan Pérez, M., Fon Abreu, Y. \& Armas Rojas, N. (2006). Intervención educativa para incrementar los conocimientos sobre bioseguridad en el personal de enfermería de una institución hospitalaria. Revista Cubana de enfermeria, 22(2).

Huanca, R., Perretta, L., Lebensohn, N., Di Tullio, L., Di Paolo, O., Pires, M., \& Quaglia, N.B. (2016). Incidencia de accidentes laborales omo potencial riesgo biológico en un hospital público de tercer nivel de atención de la provincia de Santa Fé. Revista de la Facultad de Ciencias Médicas de Córdoba, 73(2), 83-89.

Jaimes Morales, J. (2018). Sistema de gestión de seguridad y salud en el trabajo: una revisión desde los planes de emergencia. IPSA SCIENTIA. Revista Científica Multidisciplinaria, 3(1), 23-29.

Lengua, W. J., Junchaya, S. S. \& Quispe, C. S. (2014). Medidas de bioseguridad que aplica el profesional de enfermería y su relación con la exposición al riesgo laboral en el Hospital Santa María del Socorro, año 2013-2014. Revista Enfermería la Vanguardia, 2(1), 10-16.

Leitner, K. \& Resch, M.G. (2005). Do the effects of job stressors on health persist over time? A longitudinal study with observational stressor measures. Journal of Occupational Health Psychology, 10(1), 18-30.

Marrugo Ligardo, Y. (2017). Health and safety management system in Latin America: a review from the HSEQ integration. IPSA SCIENTIA. Revista Científica Multidisciplinaria, 2(1), 38-45.

Neveu, A. \& Matus, P. (2007). Residuos hospitalarios peligrosos en un centro de alta complejidad. Revista médica de Chile, $135(7), 885-895$.

IPSA SCIENTIA - Vol. 4 No $1-2019$ 


\section{Riesgo biológico generado por el manejo de residuos sólidos en un centro hospitalario}

Orozco, M. (2013). Accidentalidad por riesgo biológico en los estudiantes de enfermería de la Universidad de Ciencias Aplicadas y Ambientales U.D.C.A, Bogotá, Colombia. Revista U.D.C.A Actualidad \& Divulgación Científica, 16(1), $27-33$. https://doi.org/10.31910/rudca.v16.n1.2013.855

Palacio Angulo, J., Ahumada Villafañe, I., Orjuela, I. D., \& Posada Lopez, J. (2018). Towards a strategic epidemiology of occupational safety and health. IPSA SCIENTIA. Revista Científica Multidisciplinaria, 3(1), 30-37.

Pimentel, A., Rodríguez, V. \& Méndez, C. (2007). Remodelación del laboratorio de microbiología clínica del INER para el cumplimiento de los estándares de bioseguridad. Revista Mexicana de Ingeniería Biomédica, 28(1): 28-35.

Romero, A., Cabrera, F., Jiménez, M., Muñoz, J. \& Ávila, I. (2007). Prevalencia de accidentes biológicos por punción accidental en el Hospital Universitario Virgen de la Victoria. Rev. Nure Invest. (31): 1-10.

Suárez Pita, M., \& Junco Díaz, R. (2012). Plan institucional de manejo de los desechos sólidos, una herramienta para la gestión hospitalaria. Revista Cubana de Higiene y Epidemiología, 50(3), 415-419.

Tapias-Vargas, L.F., Torres, S.A., Tapias-Vargas, L., Santamaría, C.M., Valencia-Ángel, L.I., \& Orozco-Vargas, L.C. (2010). Accidentes biológicos en médicos residentes de Bucaramanga, Colombia. Revista Colombiana de Cirugía, 25(4), $290-299$. 
Riesgo biológico generado por el manejo de residuos sólidos en un centro hospitalario ANEXO

MATRIZ DE ELEMENTOS DE PROTECCION PERSONAL

\begin{tabular}{|c|c|c|c|c|c|c|c|c|}
\hline ACTIVIDAD & CARGO & $\begin{array}{l}\text { PARTE } \\
\text { DEL } \\
\text { CUERPO }\end{array}$ & E.P.P & ACTIVIDAD & $\begin{array}{l}\text { RIESGO } \\
\text { CUBIERTO }\end{array}$ & $\begin{array}{l}\text { ESPECIFICACIONES } \\
\text { E.P.P }\end{array}$ & CARACTERISTICAS & $\begin{array}{l}\text { MECANISMOS } \\
\text { DE ENTREGA }\end{array}$ \\
\hline \multirow{4}{*}{$\begin{array}{c}\text { LIMPIEZA } \\
\text { DE } \\
\text { GUARDIÁN / } \\
\text { CAMBIO DE } \\
\text { BOLSAS } \\
\text { ROJAS / } \\
\text { LAVADO DE } \\
\text { CAMILLAS }\end{array}$} & \multirow{4}{*}{$\begin{array}{c}\begin{array}{c}\text { Personal } \\
\text { de }\end{array} \\
\text { servicios } \\
\text { generales }\end{array}$} & Cabeza & $\begin{array}{l}\text { Gorro o cofia } \\
\text { en tela }\end{array}$ & Toda actividad & $\begin{array}{l}\text { Penetración } \\
\text { de fluidos } \\
\text { corporales, } \\
\text { residuos con } \\
\text { alta carga } \\
\text { contaminante } \\
\text { en el cuero } \\
\text { cabelludo }\end{array}$ & $\begin{array}{l}\text { cofia hecha con tela } \\
\text { antifluida que impida el } \\
\text { paso de agentes } \\
\text { biológicos }\end{array}$ & Antifluido & $\begin{array}{l}\text { reposición por } \\
\text { deterioro o } \\
\text { perdida }\end{array}$ \\
\hline & & Ojos & $\begin{array}{l}\text { Monogafas de } \\
\text { seguridad }\end{array}$ & Toda actividad & $\begin{array}{l}\text { Penetración } \\
\text { de fluidos } \\
\text { corporales, } \\
\text { residuos con } \\
\text { alta carga } \\
\text { contaminante }\end{array}$ & $\begin{array}{l}\text { Gafas de seguridad con } \\
\text { lentes de alto impacto } \\
\text { provista y que cumpla } \\
\text { con los estandares } \\
\text { OSHA N105M ANSI }\end{array}$ & $\begin{array}{l}\text { Policarbonato, } \\
\text { ventilación lateral, } \\
\text { incoloro }\end{array}$ & $\begin{array}{l}\text { reposicion por } \\
\text { deterioro o } \\
\text { perdida }\end{array}$ \\
\hline & & Nariz & Tapabocas & Toda actividad & $\begin{array}{l}\text { Impide el } \\
\text { paso malos } \\
\text { olores, } \\
\text { particulas y } \\
\text { agentes } \\
\text { biológicos } \\
\text { procedentes } \\
\text { de residuos } \\
\text { solidos }\end{array}$ & $\begin{array}{l}\text { Que cumpla con los } \\
\text { estảndares OSHA } \\
\text { N105M ANSI }\end{array}$ & $\begin{array}{l}\text { Eficiencia mínima } 95 \% \text {. } \\
\text { Para uso continuo } \\
\text { durante labores de aseo }\end{array}$ & $\begin{array}{l}\text { reposición por } \\
\text { deterioro o } \\
\text { perdida }\end{array}$ \\
\hline & & Manos & $\begin{array}{l}\text { Guantes de } \\
\text { anticortes }\end{array}$ & Toda actividad & $\begin{array}{l}\text { Protege las } \\
\text { manos de } \\
\text { cortes } \\
\text { pinchazos e } \\
\text { impide } \\
\text { contacto con } \\
\text { agentes } \\
\text { biológicos }\end{array}$ & $\begin{array}{l}\text { Que cumpla con los } \\
\text { estándares OSHA } \\
\text { N105M ANSI }\end{array}$ & $\begin{array}{l}\text { Guantes industriales de } \\
\text { caucho calibre } 35 \text {, para } \\
\text { uso continuo durante la } \\
\text { jornada de aseo }\end{array}$ & $\begin{array}{l}\text { reposición por } \\
\text { deterioro o } \\
\text { perdida }\end{array}$ \\
\hline
\end{tabular}

Fuente: Elaboración propia 